\section{Prevención de la mala alimentación en las estudiantes}

Prevention of poor nutrition in students

Artículo resultado de proyecto de investigación financiado por la Universidad de Guayaquil

Elizabeth Briggitte Quijije Logroño Medico General, Universidad de Guayaquil

https://orcid.org/0000-0001-6981$\underline{8220}$

elizabethquijije19962@gmail.com Guayaquil-Ecuador

http://www.jah-

journal.com/index.php/jah

Journal of American health, July -

December vol. 1. Num. 2-2018

Esta obra está bajo una Licencia

Creative Commons

Atribución-NoComercial-

CompartirIgual 4.0 Internacional.

RECIBIDO: 15 DE FERERO 2018

ACEPTADO: 20 DE MAYO 2018

PUBLICADO: 3 DE JULIO 2018

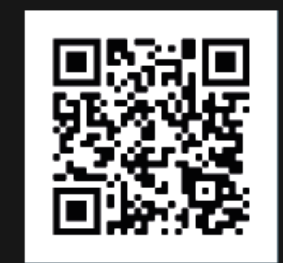

Scan this QR

code with your

smart phone or

mobile device to read more papers

\section{RESUMEN}

Mediante el presente trabajo de investigación se logra evidenciar la importancia de una correcta alimentación y su influencia en el desempeño escolar de los estudiantes de octavo año de Educación General Básica Superior, jornada matutina que pertenecen a la Unidad Educativa Fiscal Patria Ecuatoriana de la parroquia Febres Cordero en la ciudad de Guayaquil provincia del Guayas. A través de la observación se determina la importancia de una nutrición adecuada, no solo obteniendo salud, sino que también beneficios en los procesos cognitivos lo que ayudará al rendimiento académico. Esto se logró confirmar a través del método inductivo y deductivo, se desarrolló la entrevista a la autoridad educativa y las encuestas a los estudiantes, padres de familia y docentes. Mediante la interpretación de un profesional de la nutrición se elabora una guía nutricional, la cual se difunde mediante una charla de concientización a la comunidad educativa, para mejorar los hábitos alimenticios.

PALABRAS CLAVE: alimentación, desempeño escolar, guía nutricional

\section{ABSTRACT}

Through this research work, it is possible to demonstrate the importance of proper food and its influence on the school performance of eighth year students of Higher Basic General Education, morning hours that depend on the Ecuadorian Homeland Fiscal Education Unit of the parish Febres Cordero in the city of Guayaquil province of Guayas. Through observation, the importance of proper nutrition is determined, not only obtaining health, but also the benefits in cognitive processes that help academic performance. This was confirmed through the inductive 
and deductive method, the interview with the educational authority and the surveys of students, parents and teachers were analyzed. Through the interpretation of a nutrition professional, a nutritional guide is prepared, which is disseminated through an awareness talk to the educational community, to improve foodstuffs.

KEY WORD: food, school performance, nutritional guide

\section{INTRODUCCIÓN}

La mala alimentación en la adolescencia es ya un problema social, económico, salud pública considerable magnitud, tanto como para los jóvenes padres hijos familia y el ambiente cual los rodea.

Las necesidades alimenticias de los adolescentes se ven influidas por la aceleración del crecimiento que se da en la pubertad. El mayor crecimiento se produce generalmente entre los 11 y los 15 años en el caso de las chicas y entre los 13 y los 16 en el de los chicos. Los trastornos emocionales pueden afectar seriamente el equilibrio en la nutrición, provocando un consumo insuficiente o excesivo de alimentos. El estrés emocional va asociado frecuentemente a manías alimenticias y a la moda de estar delgado, además suelen saltarse comidas y desarrollar hábitos alimenticios irregulares que pueden provocar desórdenes alimenticios como la anorexia. Por otro lado, el exceso de peso y la obesidad en niños y adolescentes se ha convertido hoy en día en uno de los principales problemas nutricionales. Las causas principales son el excesivo consumo de comida basura (que tiene demasiada grasa) y los alimentos con azúcar. Finalmente, hablaremos del desayuno, esa primera comida del día que tantos adolescentes se saltan alegremente como si no tuviera importancia. Ya veremos que no es así.

Vamos a ver por qué en una etapa de crecimiento tan rápido como es la adolescencia, es tan importante alimentarse bien. Los apartados del tema son:

1. La nutrición en la adolescencia

2. Los problemas de la mala alimentación

3. ¿Y tú qué desayunas? ¡Una Coca-Cola!

4. La buena y la mala alimentación

"Que tu alimento sea tu medicina y tu medicina tu alimento» - Hipócrates -

Lo más importante de este tema:

- Comprender la importancia de alimentarse bien durante la adolescencia.

- Conocer los principales grupos de alimentos y los nutrientes que aportan a nuestro organismo.

- Analizar tus hábitos alimenticios y diferenciar los que benefician y los que perjudican tu salud. ¿CUÁLES SON LOS NUTRIENTES NECESARIOS PARA UN BUEN DESARROLLO? LOS alimentos cumplen tres funciones vitales para nuestro desarrollo, pero no todos los alimentos sirven para lo mismo. Cada uno nos aporta distintos nutrientes y el consumo equilibrado de todos ellos nos conduce a la salud y al bienestar físico y psicológico. Veamos cuáles son esas funciones: 1. Alimentos que tienen una función constructora: Son necesarios para el rápido crecimiento de los huesos y músculos y para el buen funcionamiento y reparación de todas las células y tejidos de nuestro organismo. Carne, pescado, huevos, la leche y sus derivados (queso fresco, yogurt, etc.). 2. Alimentos que tienen una función energética: Sirven para proporcionarnos la energía que necesitamos cada día para respirar, caminar,... Estos alimentos son las pastas, los cereales, 
los azúcares, los aceites y las grasas. 3. Alimentos que tienen una función reguladora: Sirven para ayudar a nuestro organismo en muchas de sus funciones desde la digestión a otros más complejos. Son las verduras y hortalizas y las frutas que contienen muchas vitaminas y minerales. También contienen mucha fibra que nos ayuda a prevenir el estreñimiento. Es necesario comer alimentos de estos tres grupos para que nuestro organismo funcione correctamente y tengamos buena salud ahora y en el futuro.

LAS MALAS DE LA PELÍCULA

Tienen muy mala fama pero lo cierto es que las grasas también son importantes para nuestra salud, por ejemplo, protegen todos nuestros órganos internos. Pero no todas las grasas son iguales, algunas son beneficiosas y otras nos perju-dican. ¿Cuáles son unas y otras? Son GRASAS BUENAS las que se encuentran en las sardinas, salmón, truchas, aceite de oliva, nueces, almendras, avellanas, aguacates. Son GRASAS PELIGROSAS aquéllas con las que se fabrican los pasteles, dulces, galletas, palmeras, mantequilla, queso curado, salchichas, hambur-guesas, carne grasa de ternera y oveja.

\section{EL SOBREPESO}

¿Cuáles son las causas del sobrepeso?

- La herencia genética de cada persona.

- El estilo de vida que llevamos.

Gran parte de lo que comemos es fácil y rápido de preparar, desde las comidas rápidas cargadas de grasa hasta los alimentos precocinados listos para calentar al microondas. Además, en la actualidad llevamos vidas más sedentarias que en ninguna otra época y los adolescentes pasan más tiempo jugando con equipos electrónicos que jugando al aire libre. La televisión es uno de los principales culpables. Los niños y adolescentes que se pasan más de cuatro horas diarias delante del televisor tienen más probabilidades de tener sobrepeso en comparación con aquellos que se pasan dos horas o menos. 2.2. LA COMIDA BASURA El director de cine estadounidense Morgan Spurlock se alimentó durante un mes entero solamente con menús de McDonald's para desayunar, comer y cenar, y se grabó a sí mismo en una película documental titulada "Super Size Me". En la película se observa el deterioro de su salud: engordó 11 kilos, le subió peligrosamente el colesterol en la sangre y aumentó su tensión arterial y su hígado adquirió el tamaño del de un alcohólico. La situación empeoró de tal manera, que "en la tercera semana pensé en abandonar el proyecto, pero finalmente aguanté". En un encuentro con varios periodistas, Spurlock señaló que "la acción educativa debe centrarse primero en los niños y luego en los adolescentes" y expresó su preocupación por los datos recientes sobre la obesidad en España: "Así comenzó todo en EEUU y ahora un 37 por ciento de los niños y adolescentes tienen exceso de grasa". Spurlock advierte que "las cadenas de comida rápida engañan a los niños y sus familias, los cuales deben reflexionar que la comida barata se acaba pagando cara a largo plazo". Los efectos de la película-documental de Spurlock fueron inmediatos, pues unos días después de ser estrenada, McDonald's anunció que dejaba de servir en EE.UU. sus raciones más grandes de bebidas y patatas fritas llamadas "Supersize". El trabajo de Spurlock no ha concluido con su filme, pues, como él mismo denuncia, "caramelos, refrescos y toda clase de comida-basura se venden en los centros escolares" donde se debe educar a los jóvenes en tener hábitos de vida saludables y no los que vayan en contra de su salud y bienestar. 2 
3. LA ADICCIÓN AL AZÚCAR El azúcar está presente en muchos productos como dulces, bebidas, helados, mermeladas, caramelos, etc. y puede producir problemas de salud e incluso adicción. El problema es que los alimentos que contienen azúcar tienen muchas calorías y nos sacian rápidamente y sin darnos cuenta dejamos de comer otros alimentos más nutritivos y así se va debitando nuestro organismo frente a las enfermedades. Según un estudio reciente hecho en Europa siete millones de niños y jóvenes acuden al colegio cada mañana sin desayunar. Este estudio también pone de manifiesto que la alimentación que realiza gran parte de los niños y adolescentes a primera hora de la mañana contiene una aportación de calorías inferior a lo recomendado. Durante el sueño, muchas funciones cerebrales "se duermen" y necesitan la energía que les dará el desayuno para poder reactivarse. Además contribuye a una mejor concentración y rendimiento en el instituto. Sin embargo, es una de las comidas que los adolescentes se saltan con más frecuencia. 3. ¿Tú qué desayunas? ¡Una Coca-Cola! Éste sí es un desayuno equilibrado (8) 1 . Disminuye nuestro sistema defensivo favoreciendo las infecciones frecuentes como catarros, forúnculos, etc. 2. Asociada con problemas al menstruar y el síndrome premenstrual. 3. Aumento de caries dentales e inflamación de las encías. 4. Anemias y deficiencias nutricionales. 5. Obesidad ya que lo que el organismo no consume en ese momento es almacenado en forma de grasa. 6. Duplica el riesgo de padecer diferentes tipos de cáncer: colon, mama, ovarios, próstata, riñón y páncreas. 7. Directamente relacionado con la aparición de la diabetes en la etapa adulta. 8. Produce problemas digestivos crónicos como malas digestiones. 9. Favorece la infección intestinal de hongos, bacterias y parásitos, a su vez estos microorganismos que viven del azúcar nos producen ansias por tomarla. El consumo excesivo de azúcar tiene serias consecuencias Subraya las palabras más importantes del cuadro anterior 6 Los elementos que debe contener el desayuno son: Leche o sus derivados: yogur, queso fresco, cuajada que combinados con los cereales aportan proteínas más calcio, hierro y zinc (tus minerales amigos). Pan, cereales en copos, gofio, tostadas: Son una buena fuente de energía y aportan hidratos de carbono a la dieta y en consecuencia la glucosa que necesita nuestro cerebro. Frutas y jugos naturales (no envasados): Para aportar las vitaminas que nuestro cuerpo necesita diariamente. Estas, también ayudarán al buen funcionamiento de intestino y órganos digestivos. Fiambres poco grasos: jamón cocido, de pollo o de pavo. ¿Y qué ocurre si no desayunamos? Algunas de las consecuencias de saltarse el desayuno son: decaimiento, falta de concentración y mal humor, debido a la falta de glucosa que es nuestro principal combustible energético. Hay que recordar que a primera hora de la mañana el organismo lleva ya 10 horas o más sin recibir ningún alimento. En edades escolares, esto condiciona el aprendizaje y produce un descenso del rendimiento, ya que la capacidad de expresión, de memoria, de creatividad y de resolución de problemas quedan afectadas. Por todo ello, se puede considerar que el desayuno es un hábito alimenticio que llega a condicionar el estado físico, psíquico y nutricional, pero no sólo de los niños y adolescentes, sino en personas de todas las edades. Una buena alimentación es uno de los pilares básicos para desarrollar y mantener una buena salud; sin embargo, en algunas etapas de la vida cobra aún más importancia. Según el informe de la Sociedad Española de Pediatría Extrahospitalaria y Atención Primaria (Sepeap), Pediatría Integral, la adolescencia conlleva un incremento de las necesidades energéticas, proteicas y de micronutrientes que supera cualquier otra época de la vida. Durante la pubertad, se adquiere el 25 por ciento de la talla adulta, se aumenta un 50 por ciento la masa esquelética, se duplica la masa muscular (sobre todo en el sexo masculino) y se 
produce un aumento del volumen sanguíneo y de los órganos internos. Todos estos cambios, tan importantes para el desarrollo y la correcta formación de una persona adulta, condicionan un aumento de las necesidades nutricionales.

Además de mantener la salud en buen estado y ser necesaria para garantizar un correcto desarrollo físico y psicológico, la alimentación durante la adolescencia puede ayudar al establecimiento de hábitos alimentarios saludables que permitan prevenir algunos problemas de salud de la edad adulta. El informe destaca que, además de ser un periodo de intensos cambios, la adolescencia es una etapa de gran riesgo nutricional, ya que aumentan mucho las necesidades, se producen cambios alimentarios importantes y pueden aparecer situaciones de riesgo; esto es debido, según el informe, a varios factores, como al sentimiento de autonomía e independencia desarrollado durante esta etapa, a las diferentes situaciones familiares, a la mayor influencia de entornos externos a la familia (como los amigos o los medios de comunicación) o a la importancia que adquiere para el adolescente el peso. Los principales hábitos alimentarios y comportamientos de riesgo que pueden desarrollar los adolescentes son:

Restricción o supresión de comidas: Según algunos estudios como el Breakfast habits, nutritional status, body weight, and academic performance in children and adolescents, publicado en la revista Journal of the Academy of Nutrition and Dietetics, entre el $30 \mathrm{y}$ el 50 por ciento de los adolescentes no desayunan o lo hacen de forma irregular e insuficiente. Esto puede provocar que presenten más dificultades en el aprendizaje y el rendimiento escolar.

Abuso de las comidas rápidas: Aunque este tipo de alimentos destaquen por ser atractivos y baratos, contienen un exceso de grasas saturadas y carbohidratos refinados, y tienen un escaso aporte de fibra, vitaminas y minerales. Además, estas comidas pueden tener un alto contenido en yodo, produciendo un excesivo aumento del acné y disfunción tiroidea. El abuso de comidas rápidas pueden provocar el desarrollo de enfermedades como la obesidad.

Ingesta entre comidas: Consumir alimentos entre las comidas produce una disminución del apetito y puede trastocar los hábitos dietéticos. En algunos casos, los alimentos que se consumen entre las comidas habituales tienen un alto contenido calórico y un bajo nivel nutritivo (bollería, dulces, snacks, refrescos, etcétera).

Seguir dietas especiales y poco equilibradas (macrobióticas, vegetarianas, etcétera): Este tipo de dietas tienen el riesgo de conducir a una carencia de nutrientes que puede afectar al correcto desarrollo y formación de la persona adulta. Hay que destacar que esto depende del tipo de dieta que se realice.

Fumar: Según el informe, las personas que fuman necesitan más del doble de vitamina C, betacarotenos, vitamina $\mathrm{E}$ y ácido fólico que el resto, ya que el tabaco interfiere en la absorción de

estos

nutrientes.

Beber alcohol: El alcohol también influye en la absorción de vitamina C, vitamina A, tiamina y ácido fólico; además, aumenta la eliminación, a través de la excreción urinaria, de zinc, magnesio y calcio, elementos necesarios para el correcto desarrollo físico y mental. 
La falta de información y el desconocimiento sobre el uso de comidas rápidas, la poca accesibilidad a los servicios de salud por barreras socioculturales, económicos y educación inadecuada entre padres e hijos contribuyen al inicio temprano de enfermedades de obedecida o anorexia, generando una enfermedad grave hasta llegar al ( abandono escolar ), decaimiento en sus estudios, problemas psicológicos.

Las probabilidades de que los y las adolescentes de 15 y 19 años mueran durante esta grave enfermedad cada año se duplican.

\section{MATERIALES Y MÉTODOS}

La consecuencia principal de seguir unos malos hábitos alimentarios es la carencia de los nutrientes necesarios para el correcto desarrollo físico y mental que se produce durante la adolescencia. Además, estos hábitos, llevados al extremo, pueden originar graves trastornos alimentarios que desemboquen en enfermedades: Según el informe de la Sociedad Española de Pediatría Extrahospitalaria y Atención Primaria, la obesidad se ha convertido en un grave problema de salud pública, debido a su alcance epidémico, su tendencia a convertirse en una enfermedad crónica y sus graves repercusiones médicas y sociales. El estudio de Alimentación y Valoración del Estado Nutricional en Adolescentes (Avena) afirma que el 25,59 por ciento de los adolescentes varones españoles sufre sobrepeso, de los cuales, el 5,68 por ciento padecería obesidad; mientras que en las mujeres, el 19,13 tiene sobrepeso, teniendo obesidad el 3,08 por ciento de ellas.

Los factores que desencadenan sobrepeso y obesidad están directamente relacionados con las dietas hipercalóricas y con poco valor nutricional, el desarrollo de malos hábitos alimentarios y el abandono de los saludables (como hacer deporte). Las consecuencias para la salud de sufrir obesidad son muy perjudiciales: desarrollo de enfermedades cardiovasculares, problemas óseos, hipertensión, cáncer de colon, síndrome de ovario poliquístico, etcétera.

Trastornos de conducta alimentaria

Esta denominación engloba a varias enfermedades que tienen rasgos comunes y que suelen ser bastante recurrentes durante la adolescencia (anorexia, bulimia y vigorexia). Según el informe, el motivo de que los trastornos de conducta alimentaria se desarrollen durante la etapa adolescente responde, entre otras cosas, al riesgo de desarrollar malos hábitos alimentarios y a la preocupación excesiva por la imagen corporal propia que puede llevar a la propia distorsión y alteración de la misma. Los cambios de peso y las conductas obsesivas que se desarrollan a causa de los trastornos pueden producir consecuencias muy graves para el organismo. La importancia de una buena alimentación pasa por la correcta ingesta de los nutrientes necesarios para favorecer el correcto desarrollo de los cambios que se producen durante la adolescencia:

Energía: La energía es la columna vertebral de todas las dietas. Se obtiene energía a partir de las proteínas, grasas y carbohidratos. Si no se cubren sus necesidades básicas, las proteínas, vitaminas y minerales no pueden llevar a cabo de forma correcta su función metabólica. Sin embargo, hay que tener en cuenta que el exceso de energía se almacena como grasa. 
Proteínas: Necesarias para el desarrollo, el crecimiento y el mantenimiento de los tejidos, ya que participan en casi todos los procesos metabólicos del organismo. Según el informe, para llevar a cabo una dieta equilibrada, es necesario que entre el 12 y el 15 por ciento de las calorías tengan su fuente en las proteínas. Las proteínas de origen animal contienen más aminoácidos que las vegetales. El exceso de proteínas puede provocar hipercalciuria (excesiva excreción

urinaria

de

calcio).

Carbohidratos: El informe de la Sepeap asegura que la mitad del aporte energético del adolescente debe provenir de los hidratos de carbono y recomienda consumir alimentos que contengan carbohidratos complejos, ya que tardan más en metabolizarse y proporcionan un aporte energético durante más tiempo. Entre los alimentos más ricos en hidratos de carbono están los vegetales, cereales, pasta, arroz y pan.

Vitaminas y minerales: La adolescencia, debido al desarrollo y rápido crecimiento que se produce, es un proceso donde las necesidades de vitaminas y minerales son mucho mayores que en etapas anteriores, como la infancia. Las sustancias más demandadas por el organismo son el hierro, calcio, Zinc, además de tiamina, riboflavina, niacina y vitaminas A, C y E. La importancia de estas vitaminas y minerales reside en que son necesarias para metabolizar el resto de nutrientes (carbohidratos, grasas y proteínas) y participan en la estructura y función celular. Los productos más ricos en estos nutrientes son la fruta, lácteos, verdura, hortalizas y pescado.

\section{RESULTADOS}

El informe establece unan serie de pautas recomendables para llevar a cabo una dieta variada y equilibrada que sirva para establecer buenos hábitos alimentarios, evite carencias de nutrientes necesarios para un correcto desarrollo y pueda prevenir enfermedades y trastornos de la conducta alimentaria.

En el desayuno se debe incluir, al menos, un producto lácteo y es recomendable tomar cereales, fruta o zumo natural; en las comidas y cenas deben estar presentes pescados, legumbres, verduras y ensaladas. Las comidas rápidas, hamburguesas y pizzas pueden tomarse, pero siempre con restricción y cuando la dieta sea equilibrada. Es recomendable hacer las patatas al horno y no fritas, comer pan integral (por su alto contenido en fibra) y primar el agua y los zumos naturales por encima de bebidas azucaradas y carbonatadas. La bollería industrial, los dulces y los fritos deben restringirse en pro de los frutos secos y las frutas deshidratadas. Es recomendable consumir entre tres y cuatro raciones diarias de leche y productos lácteos; además, los lácteos semidescremados se pueden incorporar a la dieta a partir de los 10 años. Es muy importante ingerir, al menos, tres piezas de fruta y dos raciones de verdura todos los días.

¿Que es la mala alimentación en la adolescencia?

La alimentación en la adolescencia juega un papel crucial en el desarrollo hacia la edad adulta. 
"Casi dos tercios de las muertes prematuras y un tercio de la carga de morbilidad que suceden en los adultos se atribuyen a enfermedades y/o comportamientos que comenzaron en su juventud». Así se expresa la OMS en una nota descriptiva publicada en agosto del año 2011.

En esta etapa crucial, los hábitos alimentarios que se adquieren normalmente persisten a lo largo de la vida. Y, aún en el caso de que siendo adultos cambiemos hacia un comportamiento alimentario saludable, las consecuencias de esta etapa podemos sufrirlas en forma de enfermedades crónicas, en el mejor de los casos.

No podemos olvidar que la adolescencia es un paso previo a la edad adulta y dependiendo que cómo nos cuidemos podremos disfrutar posteriormente de una vida con mayor o menor calidad.

Es una etapa de constante crecimiento en la que los tejidos requieren constantemente un aporte de nutrientes para formarse correctamente y funcionar bien.

Aparentemente, el término malnutrición asociado a esta edad, nos evoca a un adolescente flaco, que no crece bien y con poca energía.

Sin embargo, la malnutrición no solo se refleja en una pérdida de peso o falta de energía por carencia de nutrientes necesarios para la edad o con una curva inapropiada de crecimiento.

El sobrepeso y la obesidad que, hoy en día, afecta a muchos chicos del mundo y que posiblemente arrastrarán hasta la edad adulta, son malnutriciones.

Una alimentación poco saludable que no esté equilibrada y que no aporte todos los nutrientes que los chicos necesiten a pesar de que gocen de un peso y estatura normales, acarrea malnutriciones.

Los problemas en el rendimiento escolar o los fracasos universitarios pueden esconder malnutriciones, es decir, carencias de los suficientes macro y micronutrientes.

Una tendencia a conductas antisociales o depresivas pueden ser el reflejo de una alimentación inadecuada.

«Una nutrición adecuada y unos hábitos saludables de alimentación y ejercicio físico a estas edades sientan los cimientos de una buena salud en la edad adulta».

Tenemos que estar atentos y bien informados, tanto los padres como los propios adolescentes de cuáles son sus requerimientos nutricionales adaptados a las circunstancias individuales para que no existan carencias como causa de enfermedades presentes y futuras.

En la entrada Alimentación y nutrición en la adolescencia. podéis encontrar recomendaciones generales que atañen a esta edad.

Recomendaciones de la OMS:

- Administración intermitente de suplementos de ácido fólico y hierro.

- Educación sanitaria de adolescentes, padres y cuidadores sobre una alimentación sana y ejercicio físico. 
- Evaluaciones del Índice de Masa Muscular, según la edad.

EL PAPEL DE LOS PADRES Y DE LA SOCIEDAD.

Los progenitores son cruciales en la alimentación de los chicos, pero dejando a un lado algunas conductas represivas, su ejemplo es lo que guardará el adolescente como comportamiento de vida posterior.

No deberíamos exigir lo que no hacemos y, en ocasiones, sin cambiar los propios hábitos, pretendemos que los chicos se alimenten bien. Sin embargo, si el adolescente no encuentra en su propia familia hábitos que le parezcan convenientes y equilibrados que conduzcan a un buen aspecto físico como consecuencia de una buena salud, los buscará en otro lado, tendiendo a imitar o adoptar comportamiento que observa en otros lugares.

Lo anterior puede llevar a alimentaciones vegetarianas estrictas sin la debida información, obsesiones por el deporte sin un buen respaldo alimentario y nutricional, etc. Es decir, a estados de malnutrición.

La OMS publicó en el año 2014 un informe que lleva por título Salud para los Adolescentes en el Mundo, en el mismo este organismo pide una «segunda oportunidad en la segunda década». Este informe está dirigido a educadores, prestadores de servicios, promotores y a los propios jóvenes para que se actúe en las cuestiones de salud que atañen a los adolescentes.

Hay algunos aspectos de este informe que vamos a conocer porque quizás nos ayuden a ampliar espacios mentales y físicos respecto de esta etapa de la vida.

¿Cómo podemos avanzar para que los adolescentes gocen mejor salud?

Los caminos normales ya los conocemos todos, seguramente hay muchos padres preocupados por múltiples aspectos de la vida de sus hijos adolescentes, pero hay que ir «más allá»:

- Más allá de los mitos: Como por ejemplo, que está sanos y no necesitan tanta atención, que solo les preocupa su sexualidad, que no existe base científica, etc., pero todo lo anterior es erróneo. El pensamiento del adolescente va mucho más allá que el mundo de la sexualidad, necesitan atención constante para mantener su salud y no tanto centrada de forma obsesiva en su comportamiento, sino más bien rodeándoles de un mundo saludable.

- Más allá de la mortalidad: Ya que ningún adolescente debería morir por causas prevenibles o tratables y es necesario focalizar la atención en aquellas conductas que ponen en peligro su salud.

- Más allá del individuo: Las intervenciones a nivel individual no suelen ser suficientes, por ello, los cambios estructurales, ambientales y sociales son fundamentales y todo esto incluye el aumento de las medidas de apoyo a los padres y las escuelas.

- Más allá de la concentración en un solo problema: Muchos de los comportamientos y trastornos que atentan contra la salud de los adolescentes y que continuarán minando su salud en la edad adulta, así como la salud de sus hijos, tienen determinantes comunes y están concatenados, según nos dice la OMS. Las intervenciones deben estar orientadas a las causas de las múltiples conductas de riesgo. 
- Más allá de las medidas adoptadas habitualmente: Hay muchos recursos por explotar para mejorar y mantener la salud de los adolescentes.

- Más allá de las aspiraciones: Un enfoque basado en los derechos humanos hace hincapié en la actitud de los gobiernos, pues de ellos han de salir las intervenciones sociales que hagan posible algunos de los aspectos descritos.

Es un tema complejo que necesita aires nuevos, la base de un equilibrio alimentario en esta etapa de la vida, a nuestro parecer, es fundamental y es triste que la recomendación general de la OMS incluya la administración de suplementos alimentarios de hierro y ácido fólico, como consecuencia de palpables carencias, porque una alimentación nutritiva y adecuada puede cubrir completamente las necesidades de una etapa de la vida en la que búsqueda de información veraz es fundamental.

La adolescencia es un periodo del desarrollo biológico, psicológico, sexual y social inmediatamente posterior a la niñez y que comienza con la pubertad. Es un periodo vital entre la pubertad y la edad adulta, su rango de duración varía según las diferentes fuentes y opiniones médicas, científicas y psicológicas, generalmente se enmarca su inicio entre los 10 y 12 años, y su finalización a los 19 o 24.1

Anteriormente, la Organización Mundial de la Salud consideraba a adolescencia como el período comprendido entre los 10 y 19 años, comprendida dentro del período de la juventud -entre los 10 y los 24 años-. La pubertad o adolescencia inicial es la primera fase, comienza normalmente a los 10 años en las niñas y a los 11 en los niños y llega hasta los 14-15 años. La adolescencia media y tardía se extiende, hasta los 19 años. A la adolescencia le sigue la juventud plena, desde los 20 hasta los 24 años.

Este proyecto se realiza pensando en los estudiantes de la unidad educativa Patria Ecuatoriana de la ciudad de Guayaquil para que ellos sean beneficiados y para que tomen conciencia que la mala alimentación en la adolescencia es una etapa que hay que tomarla con responsabilidad y de una manera segura, los jóvenes tienen que ser orientados para que no sean juzgados por el medio que los rodea y dar a conocer que hay muchas alternativas para no llegar hacer obeso o tener una enfermedad como la anorexia.

Para prevenir mucha obesidad o anorexia en adolescentes estamos preparando esta prevención para que los adolescentes tengan una mejora en sus decisiones y puedan continuar en sus estudios y no tengan un stop en el transcurso de su año lectivo ya que tener una salud mala con obesidad o anorexia y siendo menores de edad hace que los estudiantes bajen los rendimientos académicos ya sea por muchas faltas o por la reacciones que trae estas enfermedades y les ocasionara muchas dificultades para culminar los estudios y concentrarse con toda la totalidad necesaria para nuestra educación.

Esta prevención es para que los adolescentes tengan una información adecuada de la mala alimentación a base de hechos, experiencias, sucesos e investigaciones para que ellos tomen conciencia y sus decisiones no sean erróneas ya que si es un adolescente que contiene una obesidad deberá tener la debida información de los alimentos que no le causa daño para su 
salud y los debidos métodos para no tener un obesidad o anorexia ya que en la adolescencia este es un caso que se da en muchos casos.

Los adolescentes necesitan tener información sobre las enfermedades que se dan al obtener una mala alimentación, la debilidad, las muertes que pueden causar las enfermedades de obedecida o anorexia y el riesgo que corren los y las adolescentes en su salud, ya que es grave a su edad porque es el tiempo de su desarrollo de 13-19 años de edad. A veces estas enfermedades hacen que ellos dejen sus estudios por el motivo que se sienten que no pueden dar todo su esfuerzo ya sea por desgaste físico o por la mala alimentación

La mayor razón para esta prevención es que en los últimos años ha sido alarmante el gran aumento del porcentaje de obedecida en adolescentes y muchos de los adolescentes no culminan sus estudios por el solo hecho de que su salud empeore y tendrán otras preocupaciones o obligaciones de recuperarse el $100 \%$.

Nuestro proyecto se está realizando en nuestra institución educativa Patria Ecuatoriana en las jornadas vespertina ya sea dentro de las aulas de clases en el patio central o en la sala de audiovisual ; en cual este proyecto es un proceso de tres meses en el cual vamos a ir indagando y realizando las actividades necesarias para nuestro proyecto de prevención de la mala alimentación en adolescentes, vamos a dar a conocer nuestro proyecto mediante charlas, actividades llamativas, entregando trípticos que obtengan la información necesaria para la prevención, también nos ayudaremos con objetos visuales como carteleras con el mensaje deseado .

Contamos con la predisposición de los docentes, la creatividad de cada uno de nuestros integrantes de grupo, el servicio de cada compañero y de los docentes facilitadores, también contamos con los sitios web y los textos educativos.

\section{DISCUSIÓN}

La utilidad de este proyecto es tomar en cuenta la orientación, guía, soporte y el conocimiento para todos los adolescentes de nuestra institución del significado de todos los puntos que tratemos a dar a conocer como el cual tenemos como primer punto:

La responsabilidad -. al tener una enfermedad como obesidad sin los conocimientos adecuados tanto como en la salud del adolescente, la educación, entre la sociedad y la economía.

Reducir la incidencia de nuevos casos.

Recomendar. - acciones para reducir las enfermedades como la obesidad o anorexia en estudiantes de nuestra institución.

La mala alimentación es un tema que ha cobrado mayor importancia actualmente, debido al aumento de los problemas de salud relacionados con este. La población más expuesta a sufrir enfermedades relacionadas con la mala alimentación con los jóvenes.

Los estudiantes son los más vulnerables debido al cambio en su estilo de vida.

Factores que influyen en la mala alimentación de los estudiantes 
Falta de tiempo

Los estudiantes al tener mayores responsabilidades en su estudio a veces descuidan su alimentación.

Ya sea porque tienen mucho trabajo por hacer y no comen, o no comen a sus horas.

Factores que influyen en la mala alimentación de los estudiantes.

Economía

Algunos estudiantes son de bajo nivel socioeconómico

Por lo que a veces no comen por no tener dinero para comprar.

Factores que influyen en la mala alimentación de los estudiantes.

Cambio en el estilo de vida

Algunos estudiantes pasan de un estilo de vida en el que dependen de sus padres a un estilo independiente.

Al vivir solo, tienen que aprender a valerse por sí mismos, pero algunos les resulta difícil, más en la cuestión alimentaria.

Factores que influyen en la mala alimentación de los estudiantes.

Preferencia de la comida rapida

Inactividad fisica

Habilidad para cocinar

Influencia de compañeros

Es una enfermedad muy común en personas que tienen una alimentación desequilibrada y en exceso, afectada a todos por igual, niños, jóvenes y adultos.

Las personas con obesidad están más propensos a padecer enfermedades como diabetes mellitus, hipertensión arterial y cardiopatías.

\section{ENFERMEDADES QUE SON CONSECUENCIAS DE UNA MALA ALIMENTACIÓN}

Anorexia: enfermedad psicológica

Afecta principalmente a jóvenes adolescentes, quienes evitan subir de peso porque piensan que están "gordas"

Bulimia: las personas que la padecen tienden a inducirse el vómito.

Por miedo a engordar

La población universitaria es considerada la población adulto joven clave para la promoción y prevención de salud para las futuras generaciones, por lo que es trascendental identificar la situación actual nutricional y la frecuencia de actividad física de los universitarios. 
Modificar los hábitos alimentarios y los niveles de la familia y en este caso el estudiante.

Fomentar la actividad física, realizar al menos 60 minutos de actividad.

Comer al menos cinco porciones de frutas y verduras al día.

Respetar los horarios para comer, no malpasarse.

Evitar lo más que se pueda comer alimentos chatarra y comida rápida.

Visitar a un nutriólogo para que te asesore con un plan de alimentación.

Beber abundante agua, evitas refrescos y bebida azucaradas.

\section{REFERENCIAS}

1. Barron, W. M. (s.f.). Obtenido de https://books.google.com.ec/books?id=cAYUajEZcsC\&pg=PA22\&dq=Riesgos+en+la+gestante\&hl=es\&sa=X\&ved=0ahUKEwjm3 $\mathrm{Ni} 43-$

3RAhVIB8AKHQIHC00Q6AEIGDAA\#v=onepage\&q=Riesgos\%20en\%20la\%20gestante\&f =false

2. Casanovas. (1999). Impacto del programa de rehabilitación del periné en el postparto.

3. Elembarazo.net. (2013). Obtenido de http://semanas.elembarazo.net/complicacionesfrecuentes-durante-el-embarazo.html

4. Espinoza, C. R. (2012). Psicoprofilaxis Obstetrica y Estimulacion Prenatal.

5. Fernandez, O. (2014).

6. Asosiation, american pregnancy. Efectos Secundarios Posibles Después del Aborto. s.d de 02 de 2014. https://americanpregnancy.org/es/unplanned-pregnancy/abortionside-effects/ (último acceso: 06 de 02 de 2020).

7. Burgos, Yanina Rodrigez. tipos de aborto. 02 de 09 de 2008. https://www.monografias.com/trabajos64/aborto-consecuencias/abortoconsecuencias2.shtml (último acceso: 06 de 02 de 2020).

8. fronteras, medicos sin. LAS CONSECUENCIAS DEL ABORTO NO SEGURO. s.d de 04 de 2018. https://www.msf.es/las-consecuencias-del-aborto-no-seguro (último acceso: 06 de 02 de 2020).

9. parenthood, planned. Expectativas luego de realizar un aborto en una clinica. s.f. https://www.plannedparenthood.org/es/temas-de-salud/aborto/abortos-realizadosen-una-clinica/que-puedo-esperar-despues-de-haberme-realizado-un-aborto-en-una-

10. Perez, Luis. cuidate . 29 de 05 de 2013. https://cuidateplus.marca.com/reproduccion/embarazo/diccionario/aborto.html (último acceso: 6 de 2 de 2020). 